\title{
Better cosmetic results and comparable quality of life after skin-sparing mastectomy and immediate autologous breast reconstruction compared to breast conservative treatment
}

\author{
Veronique F. Cocquyt ${ }^{a, *}$, Phillip N. Blondeel ${ }^{b}$, Herman T. Depypere ${ }^{c}$, \\ Karlien A. Van De Sijpe ${ }^{b}$, Kristof K. Daems ${ }^{d}$, Stanislas J. Monstrey ${ }^{b}$, Simon \\ J.P. Van Belle ${ }^{a}$
}

\author{
${ }^{a}$ Department of Medical Oncology, University Hospital Ghent, De Pintelaan 185, 9000 Ghent, Belgium \\ ${ }^{\mathrm{b}}$ Department of Plastic and Reconstructive Surgery, University Hospital Ghent, De Pintelaan 185, 9000 \\ Ghent, Belgium \\ ${ }^{\mathrm{C}}$ Department of Gynaecology, University Hospital Ghent, De Pintelaan 185, 9000 Ghent, Belgium \\ ${ }^{\mathrm{d}}$ Biostatician, AstraZeneca, Macclesfield, UK
}

Received 19 September 2002; accepted 16 May 2003

\author{
KEYWORDS \\ Breast cancer; Breast \\ conservative surgery; \\ Cosmetic outcome; \\ Immediate breast \\ reconstruction; \\ Preoperative \\ chemotherapy; Quality of \\ life
}

\begin{abstract}
Summary Preoperative chemotherapy (PCT) can be used in large primary breast cancer to facilitate breast conservative surgery $(\mathrm{BCS})$. Cosmetic results of BCS are influenced by the size of the residual tumour, relative to the size of the breast. After mastectomy, immediate breast reconstruction (IBR) with autologous tissue provides excellent cosmetic outcome and has proven to be safe in breast cancer patients. Besides improving overall and disease free survival, Quality of Life (QoL), body image and cosmetic outcome are also important issues after treatment for breast cancer.

In this study, Health-Related-Quality of Life (HRQL) and body image were evaluated, in patients treated with PCT, followed by BCS, or skin-sparing mastectomy (SSM) and perforator-flap breast reconstruction. Additionally, clinical observers assessed cosmetic outcome.

All participants were evaluated by the Medical Outcomes Study (MOS) 36-item Short Form Health Status Survey (SF-36, 36 items) and a study-specific questionnaire. An external panel evaluated standardised photographs of the breasts.

For all patients, norm-based scores of physical and mental health state are comparable with the general population, except for vitality (VT) score, which is somewhat lower. No significant differences can be observed between both groups. The majority of the patients were satisfied with the appearance of their breasts.

The cosmetic results, assessed by the clinical team, were significantly better for patients having IBR, compared to BCS. The mean score was 7.5/10 for IBR, versus $6.0 / 10$ for BCS $(p<0.0001)$.

Breast conserving treatment or mastectomy with reconstruction may yield comparable results of QoL, but cosmetic outcome is better after SSM and perforator-flap reconstruction. Patients must be offered both options, and clinicians should stress that both are equally effective.

(c) 2003 The British Association of Plastic Surgeons. Published by Elsevier Ltd. All rights reserved.
\end{abstract}


The surgical management of breast cancer is rapidly evolving towards less invasive procedures. Breast conservative surgery (BCS), followed with radiotherapy is equivalent to mastectomy with regard to outcome. ${ }^{1}$ Preoperative chemotherapy (PCT) is used to downstage large tumours to avoid mastectomy, and to evaluate in vivo chemosensitivity and biologic markers. ${ }^{2,3}$ However, in the event of response after PCT, it is not always clear what amount of breast tissue should be removed, in order to reach histological free margins and to avoid local recurrence (LRR). The main goal of BCS is complete removal of the tumour with adequate margins, while obtaining acceptable cosmetic effect. In instances where a large part of the breast has to be resected, followed by radiotherapy, breast conservative treatment will have an impact on cosmetic results.

New techniques of immediate breast reconstruction (IBR) with use of Deep Inferior Epigastric Perforator (DIEP) and Superior Gluteal Artery Perforator (S-GAP)-flaps provide excellent cosmetic outcome and have proven to be safe in breast cancer patients. ${ }^{4} \mathrm{~A}$ cosmetic problem involved in all types of breast reconstruction is, constructing a breast that matches colour, size, shape and symmetry of the original breast. A further evolution in perforator-flap reconstruction is the use of a free abdominal fat flap, with a small skin island for reconstruction of the nipple-areola complex. The preservation of the inframammary fold, the pectoralis major muscle and native skin envelope in skinsparing mastectomy (SSM) facilitates breast shaping during reconstruction. The use of DIEP-flaps has the advantage to preserve the whole of the rectus muscle and fascia, in contrast to transverse rectus abdominis muscle (TRAM) flap reconstruction. ${ }^{5}$ The flap is composed out of the essential elements for breast reconstruction, i.e. skin and fat. The vascularity is based on perforators of the deep inferior epigastric vessels. The advantages are easier shaping of the breast, reduced abdominal bulging, decreased postoperative pain and more predictable postoperative results. ${ }^{6}$ Contralateral mastopexy or reduction can also be performed to achieve symmetry between the breasts.

Al-Ghazal et al. demonstrated that in patients with small tumours $(<3 \mathrm{~cm})$, patient satisfaction of cosmetic outcome and psychosocial aspects are generally higher with wide local excision than with breast reconstruction or mastectomy. ${ }^{7}$ However, in this study, the majority of the patients underwent reconstruction with implants, whereas only a minority had autologous breast reconstruction ( $86 \%$ versus $14 \%)$. Therefore, we believe that these results have to be interpreted with caution, given the newer possibilities with autologous tissue transfer. No data are known about patients selfimage and cosmetic results after PCT and BCS for large tumours. As surgical techniques of BCS and of IBR have been improved over time, the impact of both procedures on patients' body image and selfesteem needs to be evaluated.

In this study, HRQL and body image were evaluated, together with a cosmetic assessment by clinical observers, in patients treated with PCT, followed by BCS, or SSM and DIEP-flap breast reconstruction.

\section{Patients and methods}

Forty-two patients who remained disease-free after surgery for primary breast cancer were included in the study. Group I consisted of 21 patients who underwent SSM and IBR with DIEP-flap for primary invasive breast cancer, ductal carcinoma in situ or as prophylactic surgery in high-risk patients. Group 2 included 21 patients who had PCT, followed by $\mathrm{BCS}$ and radiotherapy and adjuvant systemic treatment. In our PCT program, patients with tumours of at least $3 \mathrm{~cm}$ diameter on clinical examination, which were too large for upfront BCS, were included. This is in accordance with previous trials, where patients with tumours $\geq 3 \mathrm{~cm}$ were also included also in the PCT-program. ${ }^{3}$ Only downstaged patients, who reached a partial or complete clinical response to PCT, were offered breast conservative treatment. All patients had negative resection margins. In case of positive involved margins, a mastectomy was performed, but these patients were not included in the study. Patient characteristics are shown in Table 1.

At a routine postoperative follow-up visit, patients were asked to participate in this study. The Medical Outcomes Study (MOS) 36-item Short Form Health Status Survey (SF-36, 36 items) and a study-specific questionnaire of eight items were administered to all participants. Standardised photographs of the breasts were taken in three different positions: profile left and right, frontal view and oblique view left and right. A panel, consisting of a male plastic surgeon, a male gynaecologist, a female medical oncologist, a female nurse, a male psychologist and a female lay evaluated the photographs. Nine different items were assessed: contour of the breast, symmetry of shape and volume, position of the lower edge of the breast, symmetry, size and colour of the nippleareola complex, the amount and visibility of the 
scars. A score of 0-10 was given for each item, higher scores represent better results.

Demographic and clinical characteristics are shown in Table 1. Patients who underwent PCT and BCS were older than those who had IBR, and as a consequence, were also more frequently menopausal.

Women who underwent BCS had initially larger tumours than women having mastectomy and reconstruction. Patients in the former group were treated with PCT, in order to facilitate BCS. These evaluations were performed at least 1 year after completion of surgery and adjuvant treatment. Routine follow-up showed no evidence of local or distant relapse at the moment that the patients were included in the study. Mean follow-up was 42 months (range 18-86 months). Informed consent was obtained from the patients. The local Ethics Committee approved the study protocol.

\section{Statistical analysis}

The SF-36 total score and subscores were compared using a parametric test, the unpaired $t$-test. Normality was evaluated using the normal probability plot and box and whisker plots. The scores on the assessments of the photographs were compared using analysis of covariance with group and panel member as covariates. $P$-values were derived from the type III sums of squares. The study specific questionnaire items were compared using the Fisher's exact test.

\section{Results}

\section{Breast conservative treatment and mastectomy with reconstruction}

After breast reconstruction no single partial or complete flap loss was observed. Fat necrosis was seen in one patient. One patient developed a partial necrosis of the abdominal scar; another patient had a postoperative seroma. No other complications were reported.

Symmetrisation surgery of the contralateral breast was performed in four patients in the breast reconstruction group. It was not performed after BCS.

\section{SF-36 questionnaire}

The results of the SF-36 are summarised using the physical component summary (PCS) and mental component summary (MCS) scores and the eight SF-
Table 1 Demographic and clinical patient characteristics

\begin{tabular}{|c|c|c|}
\hline & $\operatorname{IBR}(n=21)$ & $\mathrm{BCS}(n=21)$ \\
\hline Mean follow-up (month) (range) & $49(18-86)$ & $36(18-49)$ \\
\hline \multicolumn{3}{|l|}{ Age (years) } \\
\hline Mean \pm SD & $46.5 \pm 8.4$ & $56 \pm 10.1$ \\
\hline Median & 48 & 55 \\
\hline Range & $30-58$ & $37-76$ \\
\hline \multicolumn{3}{|l|}{ Length (cm) } \\
\hline Mean \pm SD & $169 \pm 8.7$ & $164 \pm 6.7$ \\
\hline Median & 169 & 163 \\
\hline Range & $155-187$ & $155-180$ \\
\hline \multicolumn{3}{|l|}{ Weight before surgery (kg) } \\
\hline Mean \pm SD & $67.4 \pm 11.5$ & $71.4 \pm 13.2$ \\
\hline Median & 68 & 70 \\
\hline Range & $44-90$ & $51-98$ \\
\hline Breast circumference, mean $(\mathrm{cm})$ & 90.3 & 94.8 \\
\hline \multicolumn{3}{|l|}{ Menopausal } \\
\hline Yes & 10 & 16 \\
\hline No & 11 & 5 \\
\hline \multicolumn{3}{|l|}{ Education } \\
\hline Primary school & 1 & 5 \\
\hline Secondary school & 7 & 8 \\
\hline High school & 11 & 7 \\
\hline Missing & 2 & 1 \\
\hline \multicolumn{3}{|l|}{ Disability to work (weeks) } \\
\hline Mean & $22.9 \pm 21.3$ & $38.5 \pm 18.7$ \\
\hline Median & 14 & 52 \\
\hline Range & $0-52$ & $1-52$ \\
\hline Missing & 1 & 8 \\
\hline Previous pregnancy & 17 & 19 \\
\hline Previous breast-feeding & 7 & 6 \\
\hline \multicolumn{3}{|l|}{$T$-status } \\
\hline Prophylactic surgery $T_{0}$ & 2 & 0 \\
\hline$T_{\text {is }}$ & 4 & 0 \\
\hline$T_{1}$ & 6 & 0 \\
\hline$T_{2}$ & 7 & 17 \\
\hline$T_{3}$ & 1 & 4 \\
\hline$T_{4}$ & 0 & 0 \\
\hline Unknown & 1 & 0 \\
\hline \multicolumn{3}{|l|}{ Adjuvant treatment } \\
\hline Chemotherapy & 8 & 21 \\
\hline Tamoxifen & 5 & 11 \\
\hline Radiotherapy & 7 & 21 \\
\hline $\begin{array}{l}\text { Symmetrisation surgery of the } \\
\text { contralateral breast }\end{array}$ & 4 & 0 \\
\hline
\end{tabular}

36 subscales. $^{8}$ The PCS and MCS are scored using norm-based methods; both component scores have a mean of 50 and a SD of 10 in the general United States (US) population.

By using the SF-36 PCS, MCS and norm-based score (NBS) calculator, available on the internet at www.sf-36.com/test, comparisons of our summary measures could be made with the SF-36 profiles of the general US population. 
For all patients, NBS are comparable with the US general population. No significant differences in the PCS, MCS and in the 8 SF-36 subscales can be observed between both groups (Tables 2 and 3 ).

\section{Study-specific questionnaire}

Table 4 shows a summary of the answers on the study-specific questionnaire, dealing with body image, self-esteem and sexual attractiveness. The majority of the patients were satisfied with the appearance of their breasts. The mean aesthetic score, given by the patients themselves were comparable for both groups $7.6 / 10$ and $6.8 / 10$, respectively.

The majority of the patients reported no differences in psychosocial relationship compared to before surgery.

One-third of the patients in both groups reported more difficulties to be naked with their partner as before surgery. Half of the patients had an unchanged libido, half of them had a decreased libido, compared to before the operation. How- ever, five patients having BCS did not answer that question.

The items on psychosocial or intimate relationship did not differ among the two treatment groups.

The disability to work was larger for patients, treated with PCT and BCS, due to the fact that more frequently adjuvant chemotherapy and radiotherapy were also administered.

\section{Cosmetic assessment by the members of the panel}

Table 5 shows the cosmetic scores, given by a team of clinical observers. Two patients (one in each group) refused to be photographed, so the assessment could be done for 20 patients in each group. A significantly lower score was given to patients having breast conservative treatment, compared to breast reconstruction. This was seen for all items, except for the amount of scars. This can be attributed to an additional scar at the donor site in the lower abdomen for the preservation of the skinfat flap (Figs. 1 and 2).

Table 2 Mean scores on SF-36 physical health component scores (higher scores represent better quality of life)

\begin{tabular}{|c|c|c|c|c|}
\hline & Min-max score & $\operatorname{IBR}(n=21)$ & $\mathrm{BCS}(n=21)$ & $p$-value \\
\hline Physical functioning (PF) & $10-30$ & & & \\
\hline Mean & & 24.9 & 25.8 & $0.3703^{\mathrm{a}}$ \\
\hline SD & & 3.8 & 3.8 & \\
\hline Median & & 24.5 & 26 & \\
\hline Range & & $17-30$ & $18-30$ & \\
\hline Role physical (RP) & $4-8$ & & & \\
\hline Mean & & 6.3 & 6.9 & $0.3023^{\mathrm{a}}$ \\
\hline SD & & 1.8 & 1.5 & \\
\hline Median & & 7 & 8 & \\
\hline Range & & 4-8 & 4-8 & \\
\hline Bodily pain (BP) & $2-11$ & & & \\
\hline Mean & & 9.4 & 9.7 & $0.4930^{\mathrm{a}}$ \\
\hline SD & & 2.3 & 1.9 & \\
\hline Median & & 10 & 11 & \\
\hline Range & & 3-11 & $5-11$ & \\
\hline General health $(\mathrm{GH})$ & $5-25$ & & & \\
\hline Mean & & 18.3 & 18.3 & $0.9568^{\mathrm{a}}$ \\
\hline SD & & 4.1 & 2.8 & \\
\hline Median & & 17 & 18 & \\
\hline Range & & $9-24$ & $13-24$ & \\
\hline Total physical health $(=\mathrm{PF}+\mathrm{RP}+\mathrm{BP}+\mathrm{GH})$ & $21-74$ & & & \\
\hline Mean & & 58.8 & 60.4 & $0.4939^{\mathrm{a}}$ \\
\hline SD & & 8.8 & 6.8 & \\
\hline Median & & 56.5 & 60 & \\
\hline Range & & $45-73$ & $47-72$ & \\
\hline
\end{tabular}

a Unpaired $t$-test. 


\section{Discussion}

Local control and good cosmetic result are the main endpoints of every surgical treatment of breast cancer, independently of preoperative or postoperative treatment. However, if response to PCT is reached, it can be difficult to determine the amount of breast tissue that has to be removed in order to reach histological negative margins. Besides, removing a large part of the breast can have considerable impact on cosmetic outcome, especially in patients with small breasts. Moreover, radiotherapy is always indicated after BCS, which in itself can have an unpredictable impact on aesthetics. For the majority of tumours, radiotherapy can be avoided when a mastectomy is performed. Given the new possibilities of autologous breast reconstruction, SSM and IBR can be an alternative option for these patients.

In the knowledge that breast conservative treatment and SSM and IBR are both safe and equally effective procedures, clinicians and patients have become more interested in QoL and body image measures. ${ }^{2,4}$ Moreover, the patients' point of view is considered a further step towards a more human approach to cancer treatment. ${ }^{9}$

The type of primary surgery continues to play a role in a woman's body image, self-esteem and feelings of attractiveness, with women undergoing lumpectomy experiencing more positive outcomes than women undergoing mastectomy. ${ }^{10-12}$

In our study, it is surprising that even patients with a worse prognosis report a comparable QoL as patients with a more favourable prognosis, and moreover, QoL is mostly equal to that of the general US population. This was the case for all the subitems.

Body image and self-esteem are highly influenced by cosmetic outcome. ${ }^{13}$ In both groups, the majority of patients were satisfied with the cosmesis of their breasts. The results of the cosmetic assessment by the team of clinical observers show superior results for SSM and BRC, concerning contour, symmetry and projection of the lower margin of the breast and also concerning symmetry, size and colour of the nipple-areola complex. One drawback of the use of autologous breast reconstruction is an extra scar in the lower abdomen at the donor site of the flap. Although patient's point

Table 3 Mean scores on SF-36 mental health component scores (higher scores represent higher quality of life)

\begin{tabular}{|c|c|c|c|c|}
\hline & Min-max & $\operatorname{IBR}(n=21)$ & $\mathrm{BCS}(n=21)$ & $p$-value \\
\hline Vitality (VT) & $4-24$ & & & \\
\hline Mean & & 15.9 & 16.1 & $0.8081^{a}$ \\
\hline SD & & 4.1 & 4.1 & \\
\hline Median & & 16.5 & 17 & \\
\hline Range & & $9-23$ & $9-17$ & \\
\hline Social functioning (SF) & $2-10$ & & & \\
\hline Mean & & 8.2 & 8.6 & $0.5070^{\mathrm{a}}$ \\
\hline SD & & 1.7 & 1.6 & \\
\hline Median & & 8.5 & 9 & \\
\hline Range & & $6-10$ & $4-10$ & \\
\hline Role emotional (RE) & $3-6$ & & & \\
\hline Mean & & 5.4 & 5.1 & $0.1443^{a}$ \\
\hline SD & & 1.1 & 1.1 & \\
\hline Median & & 6 & 5 & \\
\hline Range & & $3-6$ & $3-6$ & \\
\hline Mental health $(\mathrm{MH})$ & $5-30$ & & & \\
\hline Mean & & 21.8 & 21.6 & $0.7861^{\mathrm{a}}$ \\
\hline SD & & 5.5 & 4.2 & \\
\hline Median & & 22 & 22 & \\
\hline Range & & $10-29$ & $12-28$ & \\
\hline Total mental health $(=\mathrm{VT}+\mathrm{SF}+\mathrm{RE}+\mathrm{MH})$ & $14-70$ & & & \\
\hline Mean & & 51.3 & 51.3 & $1.0000^{\mathrm{a}}$ \\
\hline SD & & 10.9 & 9.4 & \\
\hline Median & & 50.5 & 54.5 & \\
\hline Range & & $28-67$ & $30-62$ & \\
\hline
\end{tabular}

a Unpaired $t$-test. 
Table 4 Answers of patients on the study-specific questionnaire

\begin{tabular}{|c|c|c|c|}
\hline & $\operatorname{IBR}(n=21)$ & $\operatorname{BCS}(n=21)$ & $p$-value \\
\hline \multicolumn{4}{|l|}{ Items on body image } \\
\hline Satisfied with body when dressed & $9 / 21$ & $2 / 21$ & \\
\hline No altered body image as before surgery & $3 / 21$ & $13 / 21$ & \\
\hline Sad about body image & $7 / 21$ & $5 / 21$ & \\
\hline Angry about body image & $1 / 21$ & $0 / 21$ & 0.0044 \\
\hline Satisfied with appearance of breasts & $18 / 21$ & $14 / 21$ & \\
\hline Dissatisfied with appearance of breasts & $1 / 21$ & $0 / 21$ & \\
\hline Neither satisfied, nor dissatisfied & $2 / 21$ & $6 / 21$ & 0.1791 \\
\hline $\begin{array}{l}\text { Mean score concerning volume, symmetry and natural } \\
\text { aspect of breasts and nipples }(0-10)\end{array}$ & $7.6 / 10$ & $6.8 / 10$ & 0.0855 \\
\hline \multicolumn{4}{|l|}{ Items on psychosocial relationship } \\
\hline More difficulties in social relationship after surgery & $2 / 21$ & $2 / 21$ & \\
\hline No difference in social relationship & $17 / 21$ & $14 / 21$ & \\
\hline Less difficulties in social relationship & $2 / 21$ & $4 / 21$ & 0.6762 \\
\hline \multicolumn{4}{|l|}{ Items on intimate relationship } \\
\hline \multicolumn{4}{|l|}{ Difficulties to be naked with my partner as before surgery } \\
\hline Less difficulties & $1 / 21$ & $1 / 21$ & \\
\hline More & $7 / 21$ & $6 / 21$ & \\
\hline Unchanged & $13 / 21$ & $13 / 21$ & 1.0000 \\
\hline \multicolumn{4}{|l|}{ I am in the mood for having sex } \\
\hline As much as before surgery & $11 / 21$ & $10 / 21$ & \\
\hline More often & $0 / 21$ & $0 / 21$ & \\
\hline Less than before surgery & $10 / 21$ & $6 / 21$ & 0.7388 \\
\hline \multicolumn{4}{|l|}{ Items on general health } \\
\hline \multicolumn{4}{|l|}{ I worry about my health } \\
\hline More than before surgery & $7 / 21$ & $5 / 21$ & \\
\hline As much as before surgery & $6 / 21$ & $6 / 21$ & \\
\hline Less than before surgery & $8 / 21$ & $9 / 21$ & 0.9229 \\
\hline Mean mental health score $(0-10)$ & 7.4 & 7.4 & 0.7467 \\
\hline
\end{tabular}

Fisher's exact test.

Table 5 Assessment of standardised photographs by clinical observers

\begin{tabular}{llll}
\hline \multicolumn{2}{l}{ Mean score $(0-10)$} \\
\cline { 2 - 3 } & $\begin{array}{l}\text { IBR } \\
(n=20)\end{array}$ & $\begin{array}{l}\text { BCS } \\
(n=20)\end{array}$ \\
\hline Breasts & \multicolumn{3}{l}{} \\
$\quad$ Contour & 7.7 & 5.7 & $p<0.0001$ \\
Symmetry of shape & 7.6 & 5.5 & $p<0.0001$ \\
Symmetry of volume & 7.7 & 5.5 & $p<0.0001$ \\
Position lower edge & 7.9 & 5.5 & $p<0.0001$ \\
Nipple-areola & & & \\
Symmetry & 7.5 & 5.2 & $p<0.0001$ \\
Size & 7.8 & 6.5 & $p<0.0001$ \\
Colour & 7.0 & 6.6 & $p=0.0381$ \\
$\quad$ Scars & & & \\
$\quad$ Amount & 7.1 & 7.2 & $p=0.6497$ \\
$\quad$ Visibility & 7.2 & 6.7 & $p=0.0375$ \\
Global score & 7.5 & 6.0 & $p<0.0001$ \\
\hline
\end{tabular}

of view is important in aesthetic assessment of her breasts, we believe that the evaluation by an external team is somewhat more objective. Each member of the team was able to compare the photographs of different patients, before giving a final score. Moreover, the clinical observers were not influenced by emotions of cancer-related concerns or anxiety that a patient encounters when dealing with breast cancer. Each individual patient only has the capacity to observe and evaluate her own result and is often unaware of other reconstructive procedures.

Considerable adjustments and adaptation occurs during the months following surgery for breast cancer. ${ }^{14}$ Changes to the skin following radiation or the continuous healing of scars, may influence body image during the months or even years after treatment. We decided to perform the assessment at the earliest 1-year following termination of locoregional and systemic treatment. In this way, we were able to avoid the early negative influence of the recovery of surgery in the IBR group and the negative impact of adverse effects 

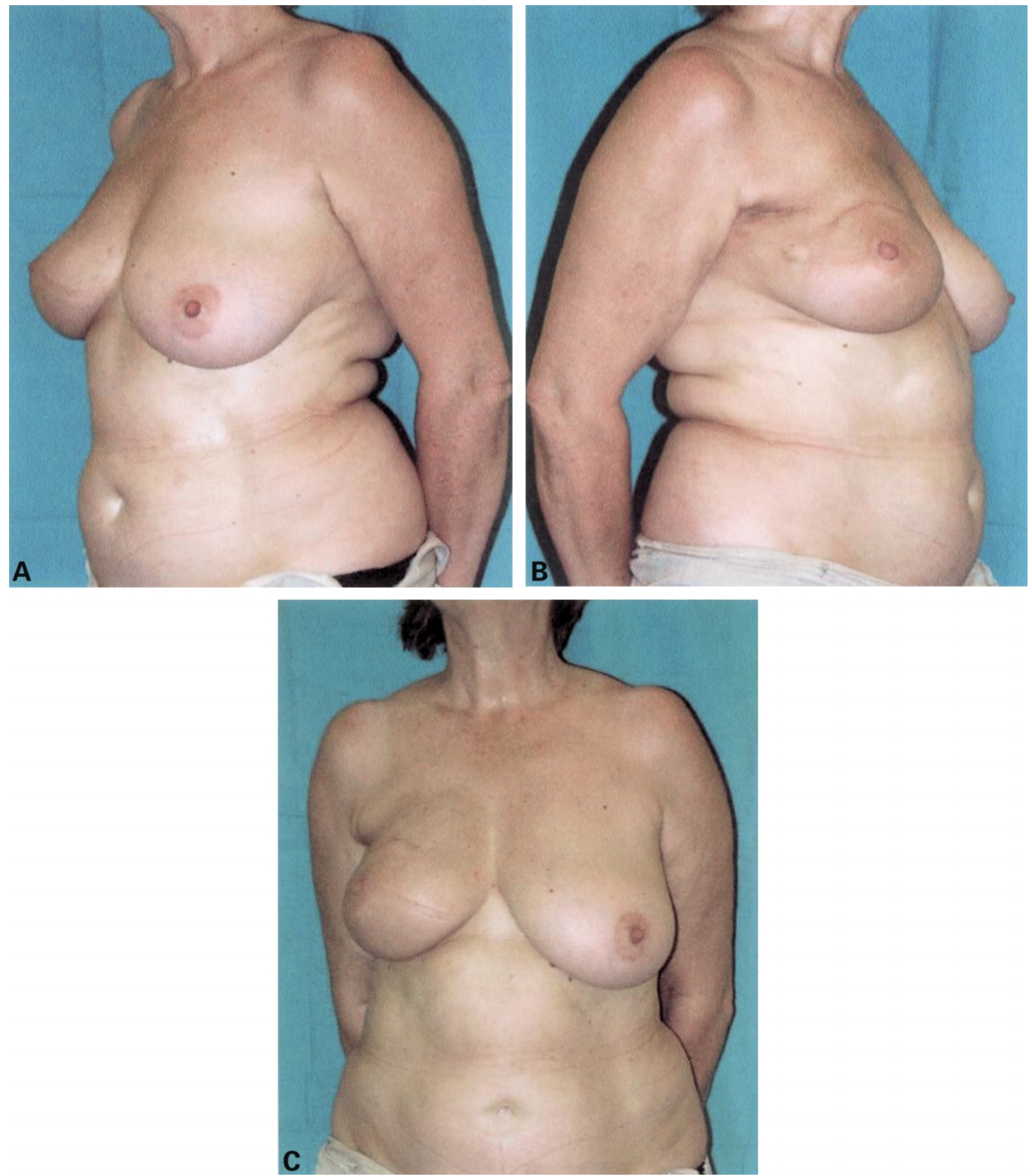

Fig. 1 A patient after breast conservative treatment in oblique view left (A), oblique view right (B) and frontal view (C).

of PCT, radiotherapy and surgery in the BCT group. The well-known late effects of radiotherapy on autologous breast reconstruction do occur predominantly within 36 months of follow-up. ${ }^{15}$ Since the mean follow-up in our patients is 42 months, we can accept that the cosmetic results will remain unchanged.

Limitations of our study focus primarily on issues of patient characteristics. In patients undergoing breast conservation, tumour volumes were larger, and all these patients were treated with chemotherapy and radiotherapy. Nevertheless, after PCT, the mean histological tumour diameter of all our patients in the neoadjuvant program was $2.3 \mathrm{~cm}$. Therefore, we do not believe that these tumours were at the upper limit of size acceptable for BCS.

Also age and menopausal status differ in both groups. The relationship between body image 

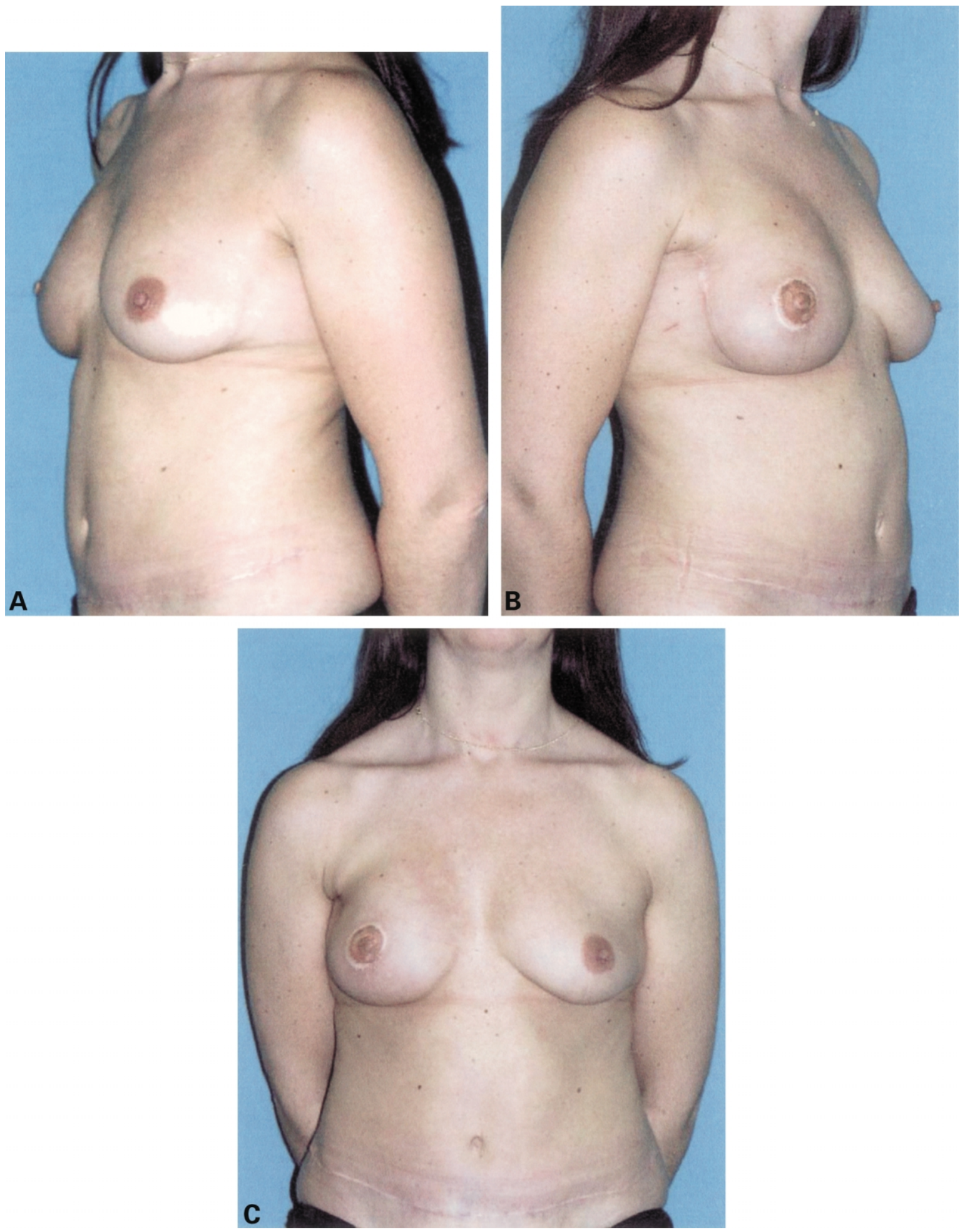

Fig. 2 A patient after autologous breast reconstruction with DIEP-flap in oblique view left (A), oblique view right (B), and frontal view (C).

satisfaction and age and marital status has been investigated, but with conflicting results. ${ }^{14}$ Therefore, we do not believe that these factors could have influenced our results.

A prospective randomised trial is needed to decrease a possible selection bias when studying psychosocial and cosmetic aspects of two different surgical procedures. However, this could be impossible to perform, as many patients would wish to choose between these options whenever possible. A 
retrospective study should always be limited by other biases, such as patient preferences, demographic differences, response to chemotherapy, location and residual size of the tumour in the breast, size of the breast, histology and grade of the tumour, nodal status, the skills of the surgeon, making a correct comparison impossible.

In conclusion, the choice of surgical procedure after PCT is predominantly, a choice of oncological safety where complete excision of all tumour tissue must be intended, but also a QoL and body image decision. Individual decision-making is critical. Patients must be offered both BCT and mastectomy with IBR and clinicians should stress that both are equally effective.

\section{References}

1. Fisher B, Redmond C, Poisson R, et al. Eight-year results of a randomized clinical trial comparing total mastectomy and lumpectomy with or without irradiation in the treatment of breast cancer. N Engl J Med 1989;320:822-8.

2. Fisher B, Bryant J, Wolmark N, et al. Effect of preoperative chemotherapy on the outcome of women with breast cancer. $J$ Clin Oncol 1998;16:2672-85.

3. Bonadonna $G$, Valagussa $P$, Brambilla $C$, et al. Primary chemotherapy in operable breast cancer: eight-year experience at the Milan Cancer Institute. J Clin Oncol 1998;16: 93-110.

4. Malata CM, McIntosh SA, Purushotham AD. Immediate breast reconstruction after mastectomy for cancer. Br J Surg 2000; 87:1455-72.
5. Blondeel PN. One hundred free DIEP flap breast reconstructions: a personal experience. Br J Plast Surg 1999;52:104-7.

6. Blondeel PN, Vanderstraeten GG, Monstrey SJ, et al. The donor site morbidity of free DIEPflaps and free TRAMflaps for breast reconstruction. Br J Plast Surg 1997;50:322-30.

7. Al-Ghazal SK, Fallowfield L, Blamey RW. Comparison of psychosocial aspects and patient satisfaction following breast conservative surgery, simple mastectomy and breast reconstruction. Eur J Cancer 2000; 36:1938-43.

8. Ware JE. The SF-36 and SF-12 health surveys: how to use them. Study guide. Boston, MA: The Health Institute/New England Medical Center; 1994.

9. Mosconi P, Colozza M, De Laurentiis M, De Placido S, Maltoni $M$. Survival, quality of life and breast cancer. Ann Oncol 2001;12:S15-S19.

10. Kiebert GM, de Haes JC, van de Velde CJ. The impact of breast conserving treatment and mastectomy on the quality of life of early-stage breast cancer patients: a review. J Clin Oncol 1991;9:1059-70.

11. Moyer A. Psychosocial outcomes of breast-conserving surgery versus mastectomy: a meta-analytic review. Health Psychol 1997; 16:284-98.

12. Rowland JH, Desmond KA, Meyerowitz BE, Belin TR, Wyatt GE, Ganz PA. Role of breast reconstructive surgery in physical and emotional outcomes among breast cancer survivors. J Natl Cancer Inst 2000;92:1422-9.

13. Al-Ghazal S, Fallowfield L, Blamey R. Does cosmetic outcome from treatment of primary breast cancer influence psychosocial morbidity? Eur J Surg Oncol 1999;25:571-3.

14. Hopwood P. The assessment of body image in cancer patients. Eur J Cancer 1993;29:276-81.

15. Tran NV, Evans GR, Kroll SS, et al. Postoperative adjuvant irradiation: effects on transverse rectus abdominis muscle flap breast reconstruction. Plast Reconstr Surg 2000;106: 313-7. 